\title{
Evaluation of the Xanthan-Based Film Incorporated with Silver Nanoparticles for Potential Application in the Nonhealing Infectious Wound
}

\author{
Jinjian Huang, ${ }^{1,2}$ Jianan Ren, ${ }^{1}$ Guopu Chen, ${ }^{1,3}$ Youming Deng, ${ }^{1,3}$ \\ Gefei Wang, ${ }^{1}$ and Xiuwen $\mathrm{Wu}^{1}$ \\ ${ }^{1}$ Department of Surgery, Jinling Hospital, Nanjing, China \\ ${ }^{2}$ Medical School of Southeast University, Nanjing, China \\ ${ }^{3}$ Medical School of Nanjing University, Nanjing, China \\ Correspondence should be addressed to Jianan Ren; jiananr@gmail.com
}

Received 29 April 2017; Accepted 18 July 2017; Published 27 August 2017

Academic Editor: Piersandro Pallavicini

Copyright (C) 2017 Jinjian Huang et al. This is an open access article distributed under the Creative Commons Attribution License, which permits unrestricted use, distribution, and reproduction in any medium, provided the original work is properly cited.

\begin{abstract}
Xanthan gum is a high molecular weight polysaccharide biocompatible to biological systems, so its products promise high potential in medicine. In this study, we crosslinked xanthan gum with citric acid to develop a transparent film for protecting the wound. Silver nanoparticles (AgNPs) are incorporated into the film to enhance the antimicrobial property of our biomaterial. This paper discussed the characteristics and manufacturing of this nanocomposite dressing. The safety of the dressing was studied using fibroblasts (L929) by the method of 3-(4,5-dimethylthiazol-2-yl)-2,5-diphenyltetrazolium bromide (MTT) assay and staining of ethidium homodimer (PI) and calcein AM. The bacterial inhibition test and application of the dressing to nonhealing wounds infected with methicillin-resistant $S$. aureus (MRSA) were performed to evaluate the antibacterial effects in vitro and in vivo, respectively. The results indicated that the dressing could restrict the formation of biofilms, reduce inflammatory reactions, and promote the angiogenesis of granulation tissues in infectious wounds. Therefore, this dressing has a great advantage over traditional clinical products especially when administered under the condition of infections or for the purpose of infection prevention.
\end{abstract}

\section{Introduction}

The treatment of nonhealing wounds is an arduous task in the case of repeated infections, severe skin damage, and altered physiological conditions such as diabetes mellitus and malnutrition [1-3]. This leads to a great financial burden for both patients and healthcare systems worldwide. Some complicated wound infections, to our great concern, are caused by virulent bacteria resistant to most of antibiotics. Staphylococcus aureus is the most common cause of these infections, and methicillin-resistant $S$. aureus (MRSA) ranks first among all drug-resistant pathogens involved in complicated skin and soft-tissue infections (cSSTI) [4-6]. Therefore, the development of an innovative dressing capable of facilitating wound observation, absorbing wound exudates, and preventing or treating the skin infections effectively is a challenging and meaningful work for the sake of accelerating the healing of complicated wounds [7].

Xanthan gum is a macromolecular polysaccharide produced mainly from sugarcane, corn, or their derivatives by Xanthomonas campestris in aerobic conditions $[8,9]$. It is extensively applied as thickener agent in the food industry, cosmetics, drilling fluids, and so on [10-12]. Hydroxyl groups in the backbone and carboxyl groups in the branched chains can be crosslinked with citric acid to form ester bonds, generating a chemical hydrogel with porous structure. The presence of hydrophilic moieties in the three-dimensional crosslinked networks of this hydrogel allows it to retain large amounts of water. The good biocompatibility of xanthan gum due to its natural origin could minimize allergic reactions against its products in human tissues [13, 14]. Moreover, the citric acid, besides being a crosslinker, can also play a role of 
an antioxidative agent to stabilize other materials, especially nanoparticles [15]. Hence, it is theoretically practical for xanthan-based hydrogel to function as the wound-protecting dressing.

In recent years, silver nanoparticles have been recognized as a powerful weapon against infectious diseases caused by various bacteria $[16,17]$. Three main methods have been reported to facilitate the synthesis of silver nanoparticles. Evaporation-condensation and laser ablation are the most common physical approaches, but consume too much energy. The most important approach for synthesis of AgNPs is chemical reduction by organic and inorganic reducing agents such as sodium citrate and sodium borohydride (NaBH4). However, the solvent contamination and uniformity of AgNP distribution are problems in the reactive process that need to be considered. The last is bio-based method, in which organisms like bacteria and plants play an important role. It is worth mentioning that this is an environmentally and economically friendly synthesis protocol [18].

Owing to the success of AgNP techniques, investigations about its potential medical practice are continuously conducted on behalf of antibacterial or anticancer agents. Taglietti et al. $[19,20]$ coated AgNPs using glutathione or pectin to modify the antibacterial effects with less cytotoxicity. Zhang et al. [21] combined silver nanoparticles with hydrogel to promote bone regeneration in infected bone defects. Basic research in oncology demonstrated that AgNPs had apoptotic effects on MCF-7 human breast cancer cells and could block the growth of prostate cancer (PC-3) cells [22, 23]. Therefore, AgNPs have recently become an indispensable component for biomaterials.

In order to explore a novel dressing to prevent infection or treat nonhealing wounds more effectively than before, the xanthan-based film was assembled with AgNPs to bring their superiority into full play. The safety and effects of this dressing for infectious wounds will be a major topic in this paper.

\section{Experiment}

2.1. Materials. The materials are as follows: commercial xanthan gum (pharmaceutical grade, viscosity of $1 \%$ solution at $20^{\circ} \mathrm{C}: 1450-2000 \mathrm{mPa} \cdot s$, Shanghai JiangLai Co. Ltd); citric acid (Sigma-Aldrich); commercial AgNPs (size: $\sim 60 \mathrm{~nm}$, Shanghai Jianglai CO. LTD); 3-(4,5-dimethylthiazolyl-2)-2,5diphenyltetrazoliumbromide (MTT) (Biotium Inc); live/dead cell staining kit (Invitrogen); ultrasonic homogenizer (model: XO-400XD; Nanjing Xianou CO. LTD); Teflon-coated plate (Nanjing Ruinike Tech Co. Ltd); UV-Visible spectrophotometry (model: UV-5200, Shanghai Metash Instrument CO. LTD); IL-6, CD68 and TNF- $\alpha$ antibodies (provided by Wuhan Goodbio Tech Co. Ltd); CD31 primer sequence (Forward: AGTAGCATCCTGGTCAACATAACA, Reverse: ATACTGTGACAACACCGTCTCTTC, designed by Primer 5 software). All other chemicals and reagents were of the highest purity grade commercially available.
2.2. Preparation of $A g$ Nanocomposite Films. 6g/L xanthan aqueous solution was mixed with AgNPs at different concentration gradients in the presence of citric acid at $300 \mathrm{mg} / \mathrm{L}$. This concentration of citric acid was chosen because it worked well for xanthan crosslinking [24]. The homogenous solution was well prepared after being thoroughly stirred overnight and blended by ultrasonic homogenizer at $50 \%$ power for 10 minutes. The solution was then poured onto Teflon-coated plates and treated in a hot air oven at $50^{\circ} \mathrm{C}$ until the films $(\sim 0.2 \mathrm{~mm})$ were casted. The casted films were directly peeled from the plates and put into the hot air oven again at $165^{\circ} \mathrm{C}$ for 7 minutes. As soon as the oven cooled down, all samples were collected and then stored in a sealed plastic bag at room temperature for further research. The working process and the product were simply illustrated in the Figure 1(a).

\subsection{Characterization of Ag Nanocomposite Films}

2.3.1. Microstructure. The micromorphology of the xanthanbased Ag film was assessed by Scanning Electron Microscopy (SEM). The films were immersed in deionized water at $37^{\circ} \mathrm{C}$ for $0.5 \mathrm{~h}$ to remove unreacted residues and then freeze-dried (in a freeze dryer). Subsequently, the freeze-dried samples were investigated by a scanning electron microscope S- 4800 at a voltage of $5 \mathrm{kV}$, through which longitudinal sections of the film were studied and images were available.

2.3.2. Identification of AgNPs. The blurry photograph of silver nanoparticles was acquired by the SEM at a high magnification of $100 \mathrm{~K}$. To obtain clear description of AgNPs, we utilized a transmission electron microscope (TEM). Samples for the transmission electron microscope were prepared by placing the original solution on a carbon-coated copper grid. The grid was air-dried and then reacted at $165^{\circ} \mathrm{C}$ for 7 minutes before TEM scanning.

2.3.3. Gel Content and Swelling Ratio. Gel content and hydrogel swelling ratio are determined by the formula as follows:

$$
\begin{aligned}
\text { Gel content }(\%) & =\frac{M_{\text {driedgel }}}{M_{\text {pol }}} \times 100 ; \\
\text { Swelling ratio }(\%) & =\frac{\left(M_{\text {swollengel }}-M_{\text {driedgel }}\right)}{M_{\text {driedgel }}} \times 100 .
\end{aligned}
$$

$M_{\text {pol }}$ represents the initial mass of polymer. $M_{\text {driedgel }}$ and $M_{\text {swollengel }}$ stand for the mass of dried hydrogel and swollen hydrogel at equilibrium, respectively.

The study of the gel content started with dried films, which then were soaked in an excess of PBS buffer for 48 hours to rinse away all unreacted reagents and by-products. Subsequently, these wet samples were removed from PBS buffer and dried under vacuum until the weight was constant. With regard to measurement of swelling ratio, several freezedried hydrogels were prepared first and then immersed in PBS buffer. At each setting time, they were removed and the surface moisture was immediately wiped off using tissue 


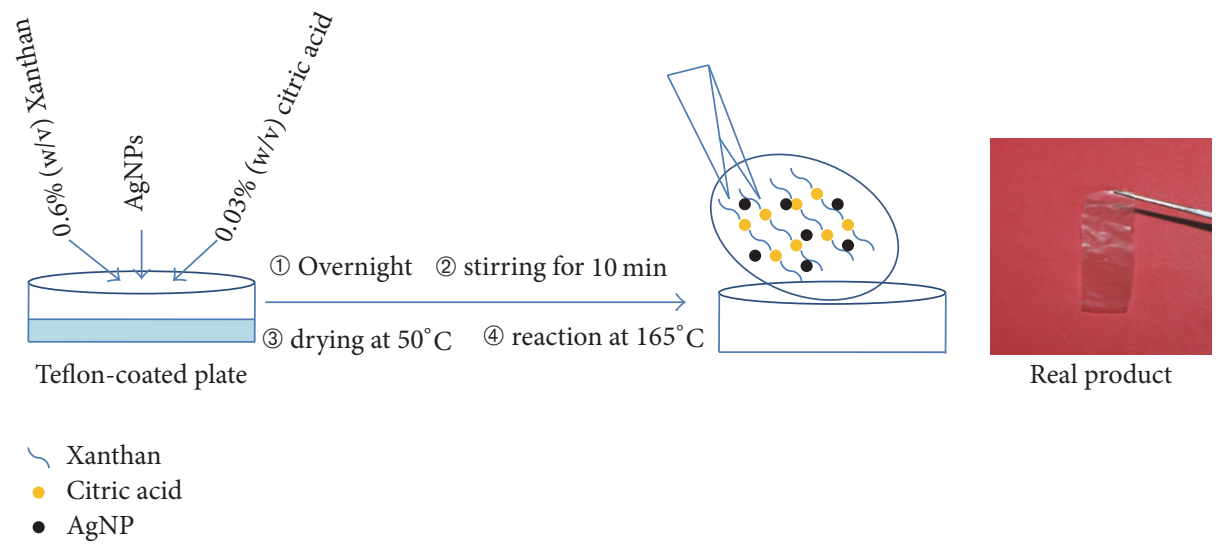

(a)

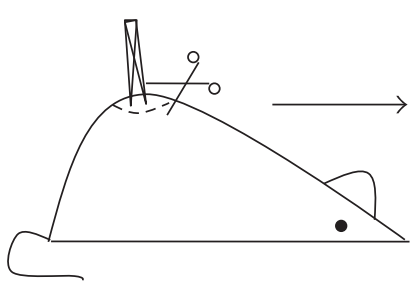

(1)

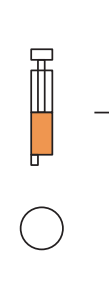

(2)

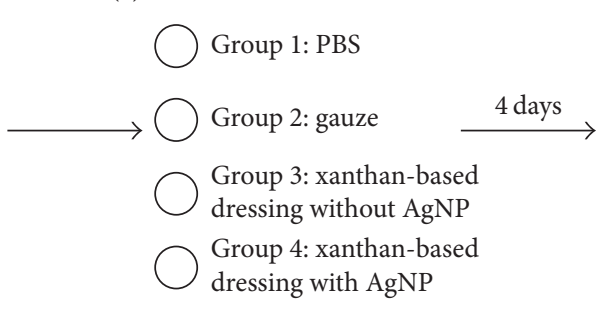

(3)

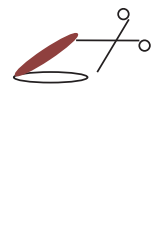

(4) and

represent the wound,

represent new granulation tissue

(b)

Figure 1: (a) The scheme of the Ag nanocomposite film manufacture; (b) the flow chart of making infectious wound model and different interventions.

paper. After being weighed, they were returned to the PBS buffer again. This process was repeated until reaching the equilibrium. Gel content and swelling ratio were calculated according to the equation above.

2.3.4. AgNP Releasing Test. The concentration of AgNPs diffused from the xanthan-based dressing was detected using UV-Visible spectrophotometry. Briefly, the AgNPs were added to PBS solution at the concentration of $2 \mu \mathrm{g} / \mathrm{ml}$, $4 \mu \mathrm{g} / \mathrm{ml}, 6 \mu \mathrm{g} / \mathrm{ml}, 8 \mu \mathrm{g} / \mathrm{ml}$, and $10 \mu \mathrm{g} / \mathrm{ml}$. These standard solutions were established after blend for 2 minutes using an ultrasonic homogenizer. The relation of the different AgNP concentrations and their relevant absorbance was automatically drawn by UV-Visible spectrophotometry at the absorption peak of $400 \mathrm{~nm}[25,26]$. The nanocomposite films made from $4 \mathrm{ml}$ homogenous xanthan solution with AgNPs at $80 \mathrm{mg} / \mathrm{L}$ were immersed in test tubes with $4 \mathrm{ml}$ PBS solution. At different points, all the PBS solution was taken away to measure its absorbance and then poured back into the tube. The ratio of AgNP release was calculated depending on the formula below:

$$
\text { AgNP releasing ratio }=\frac{\left(C_{\mathrm{AgNP}} \times V_{\text {remove }}\right)}{M_{\text {total }}} .
$$

$C_{\mathrm{AgNP}}$ is the concentration of AgNPs. $V_{\text {remove }}$ refers to the volume of PBS solution removed from the test tube. $M_{\text {total }}$ is the total mass of AgNPs in the nanocomposite film and the value of the $M_{\text {total }}$ is exactly $0.32 \mathrm{mg}$ after calculation in this experiment.

2.4. Cell Viability Assay. The MTT assay was used to investigate the cell viability. The films with different concentrations of AgNPs were cut into round pieces with a radius of $1.5 \mathrm{~cm}$. After sterilizing under UV light, these round dressings were transferred to glass plates and $2 \mathrm{ml}$ medium with fibroblasts (L929) was seeded. At the end of cell culture for 24, 48, or 72 hours, respectively, MTT $(5 \mathrm{mg} / \mathrm{mL})$ reagent was added and further incubated for 4 hours. Afterwards, the formazan salt was dissolved with $0.2 \mathrm{~mL}$ DMSO. As soon as thoroughly dissolved, the solution was added to a new plate and measured with a microplate Spectrophotometer at $570 \mathrm{~nm}(n=3)$.

To further confirm the cell viability and cell morphology, live/dead staining was performed at 24 hours by adding $2 \mathrm{mM}$ calcein AM and $4 \mathrm{mM}$ ethidium homodimer to the dressing. After washing with PBS buffer, the images were documented by an inverted fluorescence microscope.

2.5. Antibacterial Test In Vitro. The clinic sample of Staphylococcus aureus was acquired from a skin-infected patient. The minimal inhibitory concentration (MIC) of oxacillin measured with a broth dilution method was larger than $4 \mu \mathrm{g} / \mathrm{ml}$, which proved these bacteria to be MRSA. Further 
investigation in the department of laboratory medicine using PCR technology indicated the mecA gene of penicillin binding protein was positive [27]. Bacterial suspension was prepared by adding the $S$. aureus isolates until the turbidity was around 0.5 based on McFarland standards. Subsequently, isolates of $S$. aureus were inoculated onto mannitol salt agar in the condition of $37^{\circ} \mathrm{C}$. Simultaneously, the xanthan-based dressing loaded with or without AgNPs was put onto the agar surface. After 24 hours, the results were recorded by camera.

2.6. Acute Wound Infection Model for Assessing Efficiency of the Dressing. Twenty-four adult male Sprague-Dawley rats (weight ranges from $180 \mathrm{~g}$ to $220 \mathrm{~g}$, Mergene Co. Ltd) were used for this experiment. All the rats were raised at the temperature of $25^{\circ} \mathrm{C}$ and the relative humidity of 50-60\% in regular day-and-night cycles and allowed free access to food and water. All the research protocols were approved by Animal Investigation Ethics Committee of Jinling Hospital and conducted in accordance with international standards on animal welfare.

All the rats got general anesthesia through intraperitoneal injections of $10 \%$ chloral hydrate at $0.4 \mathrm{ml} / 100 \mathrm{~g}$ and were then randomly divided into four groups with six per group. The infectious wound was created by removing circular skin at $1 \mathrm{~cm}$ in diameter on the back of these animals and injecting $0.1 \mathrm{ml}$ bacterial suspension onto the defected area. After that, different interventions were performed on the wound. Specifically, each group received PBS solution, gauze, simple xanthan-based dressing, and xanthan-based dressing with AgNPs, respectively. All the rats were put back into their cages and allowed free access to water and food. Four days later, these animals were sacrificed and fresh granulation tissues were excised completely. Promptly, one-half of granulation tissues was immersed in $10 \%$ neutral formaldehyde for the histological staining and the other half was stored in $-80^{\circ} \mathrm{C}$ for the qPCR. All the procedures were briefly introduced in Figure 1(b).

The samples were taken out of formaldehyde, dehydrated, and subsequently embedded in paraffin. Serial sections with $5 \mu \mathrm{m}$ thickness for each granulation tissue were made with a microtome. These sections were stained with H\&E and Masson trichrome staining. After drying overnight, these slides were microscopically examined and then photographed. IL6 , TNF- $\alpha$, and macrophages were analysed by immunohistochemical staining of IL-6, TNF- $\alpha$, and CD68, respectively, according to standard protocols. Three images each slide were acquired at 200x magnification randomly for measuring their integral optical density (IOD) using image-pro plus 6.0 software. SPSS16.0 was used to analyse the data via One-Way ANOVA test.

\section{Results}

\subsection{Physical Properties of Ag Nanocomposite Films}

3.1.1. Micromorphology. SEM photographs of the freezedried dressing were investigated to observe the microstructure and assess its porous property. In our study, the pore size was distributed evenly around $100 \mu \mathrm{m}$. On the surface of pores, we found that the AgNPs were of round shape and welldispersed without large aggregation (Figure 2(a)). The clear photographs taken by the TEM also verified it (Figure 2(b)).

3.1.2. Swelling Ratio. According to formula (1), the gel content was calculated to $75 \%$ on average. In addition, the swelling curve (Figure 2(c)) demonstrated that this film was able to take in large amounts of PBS solution as fifteen times as its dry weight.

3.1.3. AgNP Releasing Curve. The law of AgNP release determined the antibacterial potency of the dressing. UV-Visible spectrophotometry was employed to establish the equation as follows:

$$
C=3.194 \times A+0.051 \quad(r=1.000),
$$

where $C$ and $A$ represented the concentration of AgNPs and relevant absorbance at $400 \mathrm{~nm}$, respectively. It was shown in Figure 2(d) that this nanocomposite film could prevent the outburst of AgNP release and maintain the antibacterial ability for 24 hours.

3.2. Cytotoxicity Evaluation In Vitro. The nontoxicity of medical biomaterials is always the first consideration. The safety of the film was examined with a cell viability test by MTT assay. As shown in the Figure 3, the simple xanthanbased film was safe enough for fibroblasts while the Ag nanocomposite film showed varied cytotoxicity associated with the concentration of AgNPs. But when AgNPs were less than $10 \mu \mathrm{g} / \mathrm{ml}$, this biomaterial was nontoxic to fibroblasts in vitro. Therefore, we chose this AgNP concentration for further research.

3.3. Antibacterial Activity In Vitro. The bacteriostatic ring was a direct evidence of antibacterial property. In our study, we found that simple xanthan-based film could form a small inhibition zone (Figure 4(a)). The potential reason might be the citric acid free from the film during the degradation period to function as an antibacterial agent. When AgNPs were loaded, the nanocomposite film generated a larger inhibition zone to the MRSA but did not do harm to the fibroblasts (Figure 4(b)).

3.4. Results of Histological Evaluation. During the experiment, all rats showed no adverse reactions and the symptoms of toxicity. The rats exhibited free movement and normal behavior and were sensitive to light, sound, and other stimulations. The infectious wounds in each group were photographed on day 0 , day 1 , and day 4 (Figure S1 in Supplementary Material available online at https://doi.org/10.1155/2017/6802397). The rats in the Ag nanocomposite film group showed the higher healing rate for their infectious wounds.

3.4.1. Biofilm and Collagen Fibers. The tissue samples were available to observe the condition of biofilm and wound 


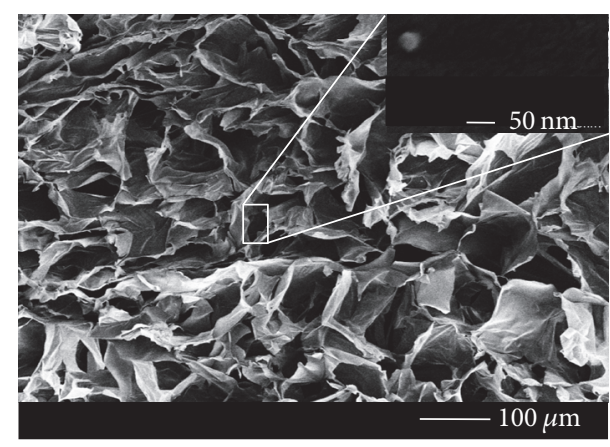

(a)

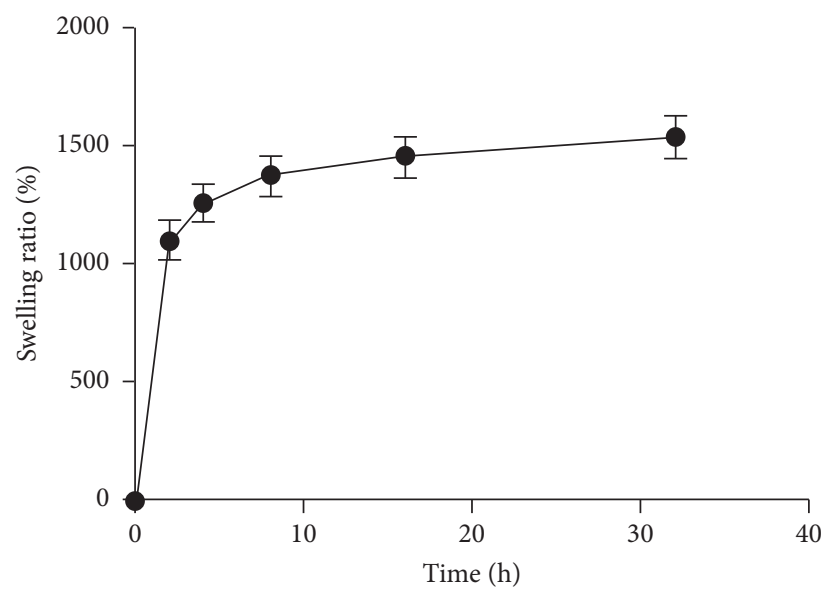

(c)

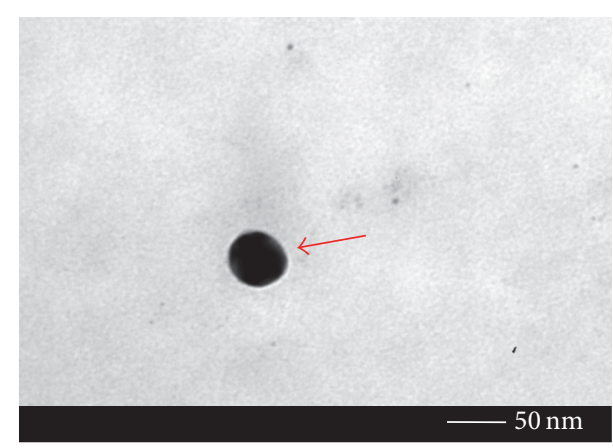

(b)

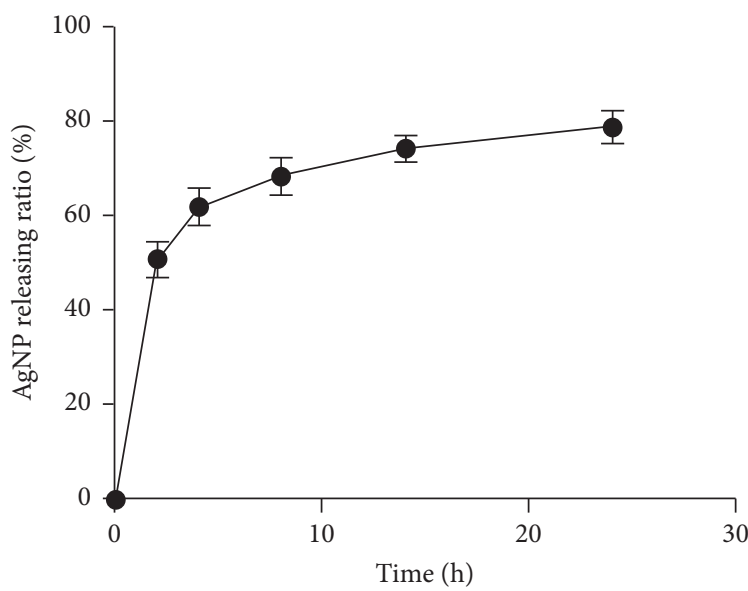

(d)

FIgURE 2: (a) The porous structure under the SEM scanning and the AgNP (inset in the corner); (b) the clear photograph of the AgNP under the TEM scanning (red arrow: AgNP); (c) swelling curve; (d) AgNP releasing curve.

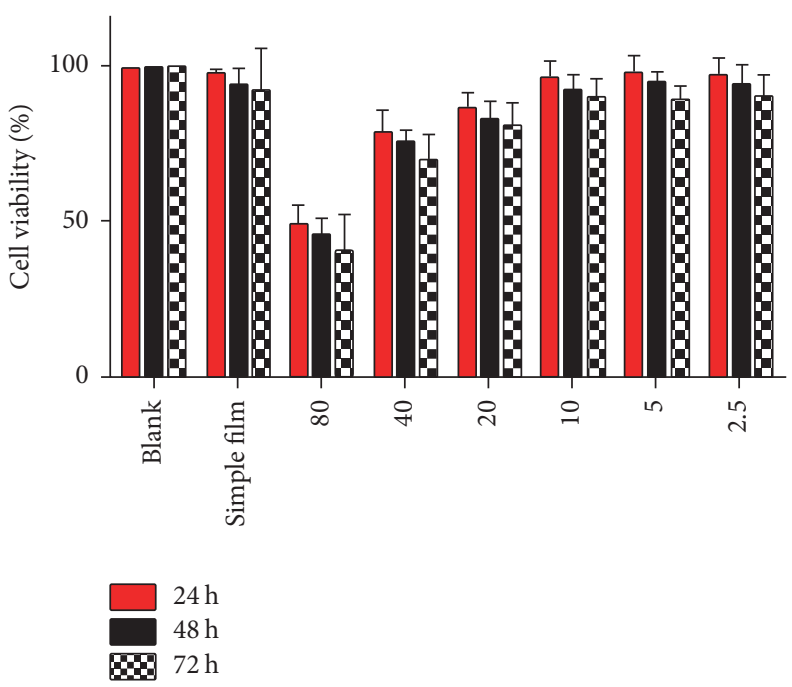

FIGURE 3: MTT results after coculture of fibroblasts and different concentrations ( $\mu \mathrm{g} / \mathrm{ml})$ of AgNPs used for making the nanocomposite films.

healing by $\mathrm{H} \& \mathrm{E}$ and Masson trichrome staining. Biofilm formations were clearly presented in the PBS group (Figure 5(a)) and the gauze group (Figure 5(b)) and less distinct in the simple film group (Figure 5(c)). However, we hardly observed the biofilm on the surface of regenerative granulation tissue in the Ag nanocomposite film group (Figure 5(d)), in which the density of collagen fibers was also the largest (Figure 6). 


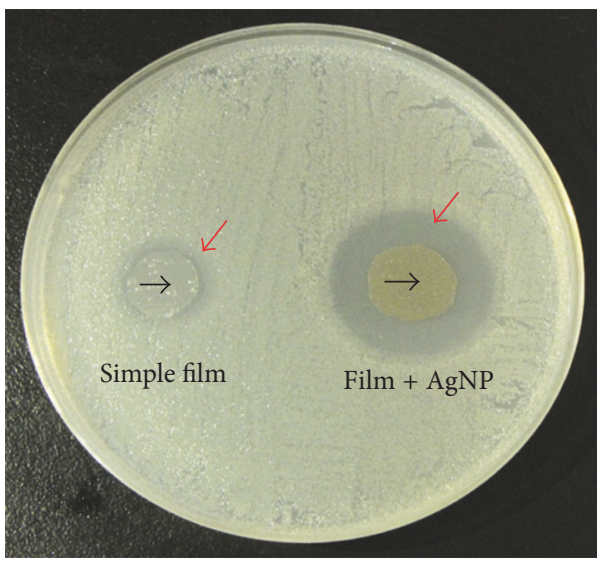

(a)

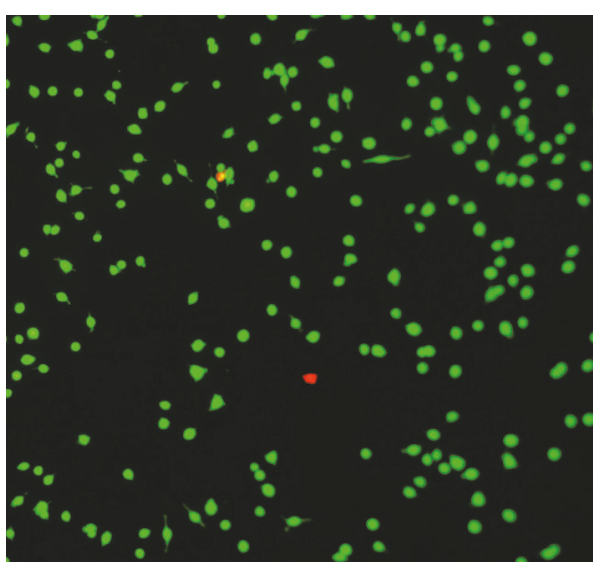

(b)

FIGURE 4: (a) The result of the antibacterial test (black arrows: films, red arrows: inhibition zone); (b) the result of the live/dead staining assay (green: live cells, red: dead cells).

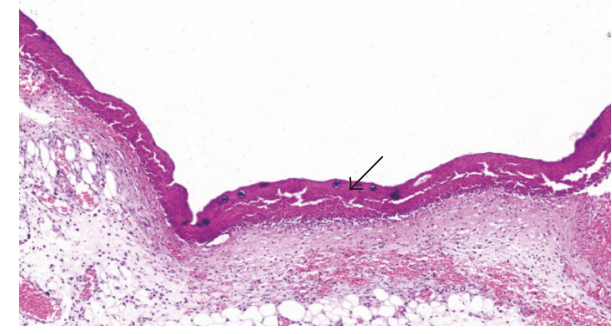

(a)

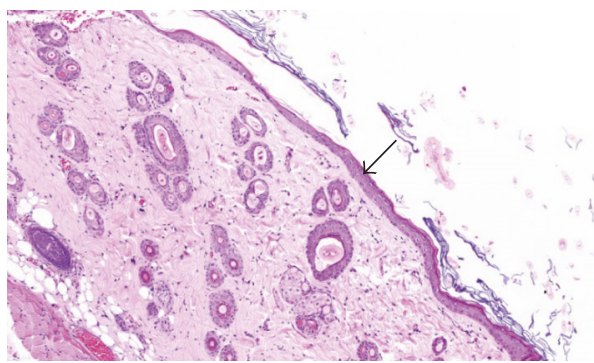

(c)

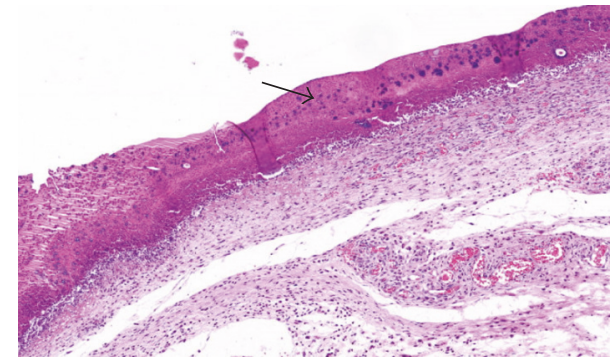

(b)

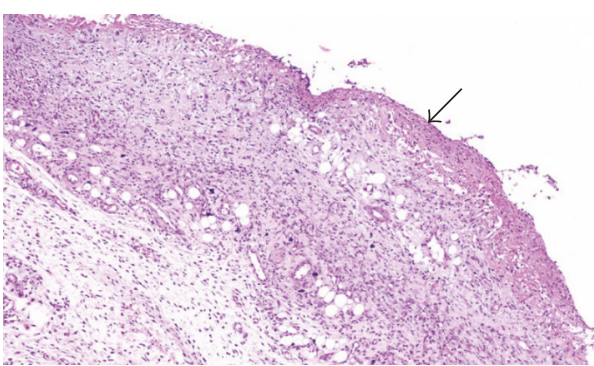

(d)

FIGURE 5: Biofilm formation (black arrows: biofilm), 100x. (a) PBS group; (b) gauze group; (c) simple film group; (d) film + AgNP group.

3.4.2. Inflammation. The inflammatory reaction deteriorated wound healing triggered by bacterial infections or toxicity of materials in vivo. Therefore, inflammatory cytokines and cells were measured to evaluate this aspect. In our study, we marked IL-6 (Figure 7(a), Figure S2), TNF- $\alpha$ (Figure 7(b), Figure S3), and macrophages (Figure 7(c), Figure S4), which revealed that the level of inflammatory cytokines and cells was the lowest in the AgNP nanocomposite film group compared with other groups.

3.4.3. Angiogenesis. Angiogenesis is the key factor of granulation tissue regeneration. CD31, a biomarker of vascular endothelial cells, was employed to investigate the transcription activity of angiogenic effects. As shown in the
Figure $7(\mathrm{~d})$, the relative mRNA expression was upregulated in the AgNP nanocomposite film group compared to other groups.

\section{Discussion}

In this study, we developed a novel Ag nanocomposite dressing using xanthan, citric acid, and AgNPs under high temperature for crosslinking. This composite material was proved to prevent the outburst of AgNP release and absorb large amounts of liquids. Moreover, experiments in vivo revealed that this dressing could block the biofilm formations in the acute infectious wound and promote the angiogenesis of fresh granulation tissues. 


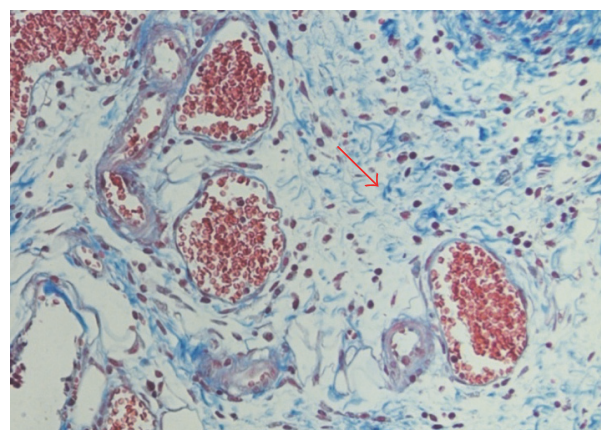

(a)

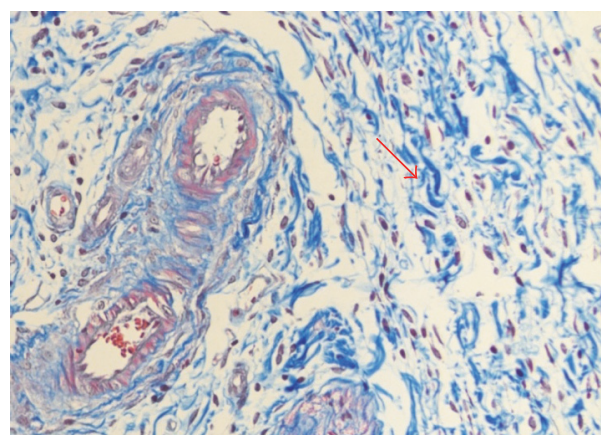

(c)

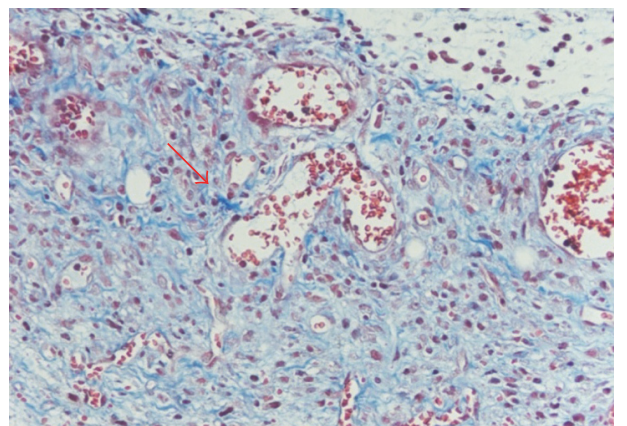

(b)

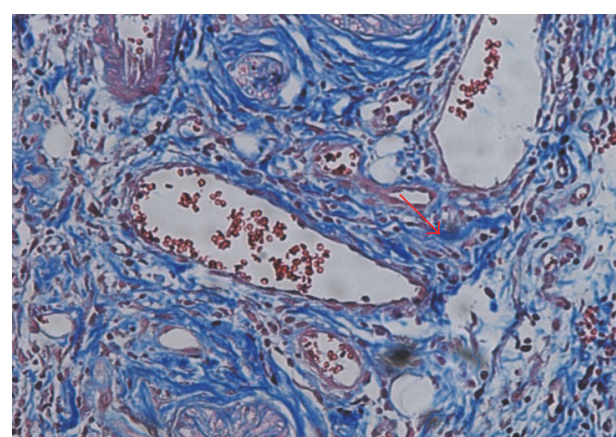

(d)

FIGURE 6: Results of Masson trichrome staining (red arrows: collagen fibers), 200x. (a) PBS group; (b) gauze group; (c) simple film group; (d) film + AgNP group.

Recently, it has been reported that the outstanding antibacterial property should be attributed to the Ag+ released from the AgNPs [28]. Chelation of Ag+ is believed to cause the collapse of bacterial membrane and prohibit DNA unwinding [29] while the AgNPs may result in the cell damage in the way of oxidative stress [30], but it is dose-dependent. The results in our experiment showed that the AgNPs would be released from the xanthan-based film gradually so that it could prevent the acute cytotoxicity as well as increase the action time of this nanocomposite material. In addition, our study verified that the concentration of AgNPs at $10 \mu \mathrm{g} / \mathrm{ml}$ could inhibit growth of MRSA but not affect fibroblasts. The size and coating of AgNPs can also influence their roles [31]. Paredes et al. [32] synthesized spherical silver nanoparticles with an average size of $55 \mathrm{~nm}$ and proved that its MIC concentration was around $0.25 \mu \mathrm{g} / \mathrm{ml}$ for $S$. aureus. The concentration of AgNPs used in our in vitro and in vivo experiments was larger than that reported in literature.

Additionally, the xanthan-based film provides a tissue scaffold for wound healing. Via the SEM, we found that this film contained a porous structure. The porosity is an important parameter for wound sites to achieve adequate nutrition, as well as gas and fluid exchange with the outer environment [33]. Besides, macroporous size is beneficial for cell regeneration and interaction [34]. Therefore, the films of around $100 \mathrm{~mm}$ in the pore diameter can conduce to the protection and repairment of infected wounds.

The biofilm lifecycle consists of four stages: attachment, growth, maturation, and detachment [35]. The mature biofilm is recognized as an early indicator for the risk of tissue infection and wound chronicity by means of inducing a host response. Neutrophils and other immune cells can not physically reach bacteria to engulf them since they are trapped within the biofilm architecture, consequently causing cell death [36]. The enzymes and DNA released from dead cells, in combination with bacteria's products, generate a serious inflammatory reaction. This is a primary mechanism accounting for the biofilm-caused impairment of normal wound healing. Our Ag nanocomposite films were able to destroy the MRSA and block the attachment stage and growth stage of biofilm formations for infectious wounds. It was demonstrated by the immunohistochemical analysis that the inflammatory cytokines and cells like IL-6, TNF$\alpha$, and macrophages were downregulated after application of the nanocomposite materials. Besides, CD31 transcriptive activity was elevated so that the wound could obtain more blood supply and nutrition for generation of collagen fibers and growth of granulation tissues.

Wound healing involves three classic stages: the inflammatory stage, tissue regenerative stage, and remodeling stage [37]. As a preliminary research, the limitations must be addressed. Our focus was mainly on inflammatory response, collagen fiber regeneration, and angiogenesis at the early stage but ignored to study the material's influence on the remodeling stage such as the pigment deposition and scar formation. So further researches are required to evaluate these aspects. 


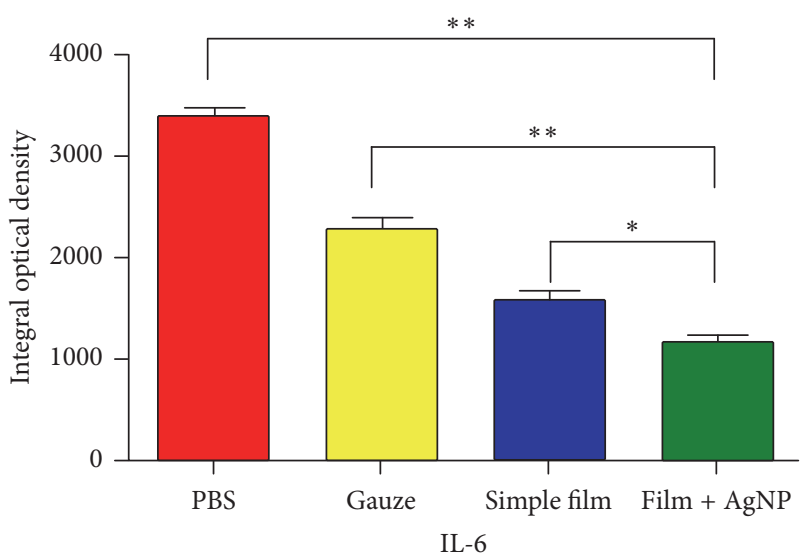

(a)

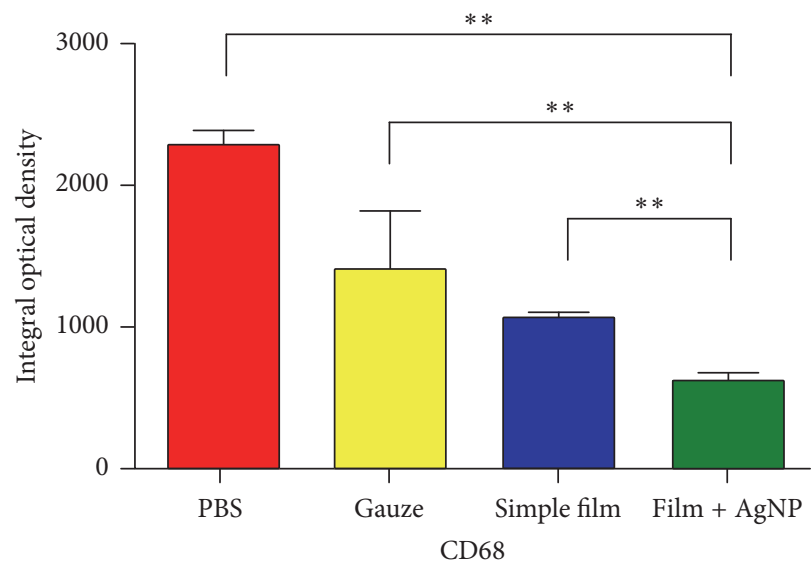

(c)

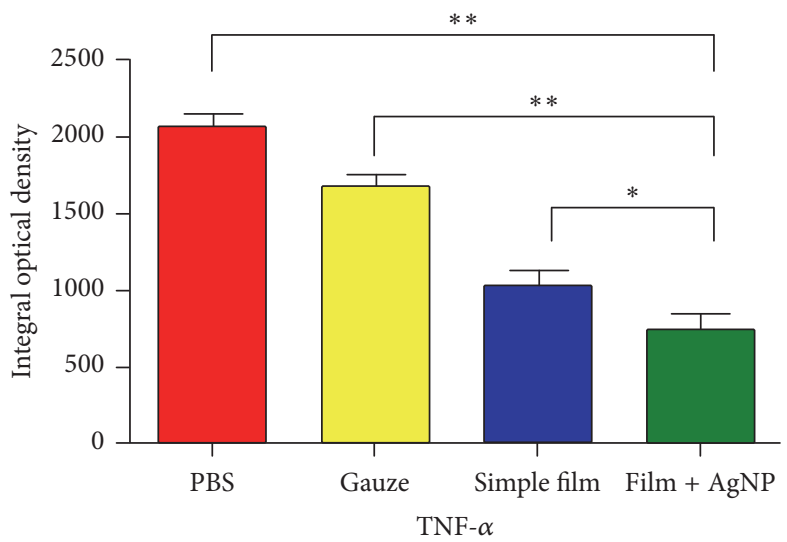

(b)

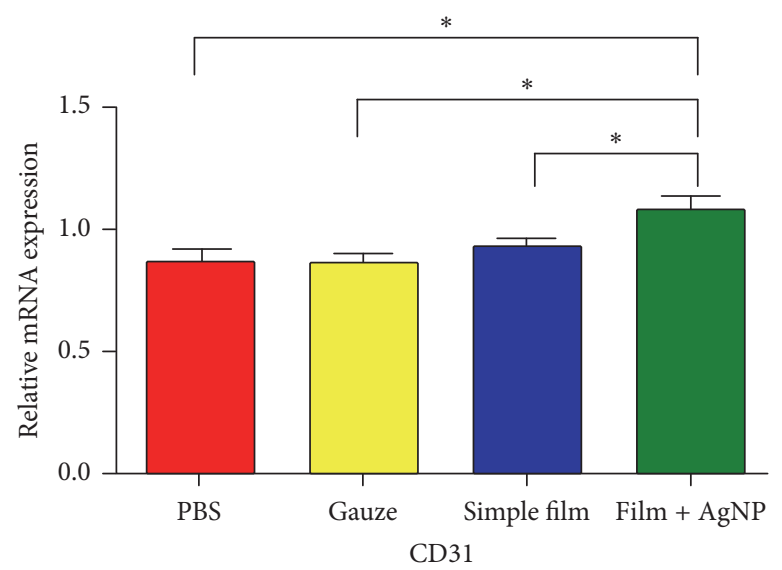

(d)

FIGURE 7: (a) IOD value of IL-6 immunohistochemical analysis; (b) IOD value of TNF- $\alpha$ immunohistochemical analysis; (c) IOD value of CD68 immunohistochemical analysis; (d) relative mRNA expression of CD31. IOD (integral optical density), ${ }^{*} P<0.05,{ }^{* *} P<0.01$.

\section{Conclusion}

Biofilm formation is a worldwide issue, leading to delayed wound healing. Our work was to incorporate the xanthanbased film with sliver nanoparticles to exert its antibacterial ability. The results demonstrated that the newly developed dressing could protect the wound against the damage of biofilms and take in large amounts of exudates. It was showed by histological examination that this composite material was able to alleviate inflammatory reactions and promote the growth of granulation tissues. In a word, this dressing has a broad prospect in the treatment of wound infections.

\section{Conflicts of Interest}

The authors declared no potential conflicts of interest with respect to the research, authorship, and publication of this article.

\section{Acknowledgments}

The authors are grateful to Dr. Luise Brakert in the University Medical Center of Hamburg-Eppendorf for helping them to organize their manuscript and improve their scientific language. This research was funded by the National Natural Science Foundation of China (Grant no. 81571881) and Key Project of Jiangsu Social Development (Grant no. BE2016752).

\section{References}

[1] A. Lemaignen, G. Birgand, W. Ghodhbane et al., "Sternal wound infection after cardiac surgery: incidence and risk factors according to clinical presentation," Clinical Microbiology and Infection, vol. 21, no. 7, pp. 674-e11, 2015.

[2] D. G. Greenhalgh and R. L. Gamelli, "Is impaired wound healing caused by infection or nutritional depletion?" Surgery, vol. 102, no. 2, pp. 306-312, 1987.

[3] T. K. Hunt and H. W. Hopf, "Wound healing and wound infection: what surgeons and anesthesiologists can do," Surgical Clinics of North America, vol. 77, no. 3, pp. 587-606, 1997.

[4] C. Ghosh, G. B. Manjunath, M. M. Konai et al., "Aryl-alkyllysines: Agents that kill planktonic cells, persister cells, biofilms of mrsa and protect mice from skin-infection," PLOS ONE, vol. 10, no. 12, Article ID e0144094, 2015.

[5] A. Russo, E. Concia, F. Cristini et al., "Current and future trends in antibiotic therapy of acute bacterial skin and skin-structure 
infections," Clinical Microbiology and Infection, vol. 22, pp. S27S36, 2016.

[6] L. J. Bessa, P. Fazii, M. Di Giulio, and L. Cellini, "Bacterial isolates from infected wounds and their antibiotic susceptibility pattern: some remarks about wound infection," International Wound Journal, 2013.

[7] C. Ghobril and M. W. Grinstaff, "The chemistry and engineering of polymeric hydrogel adhesives for wound closure: A tutorial," Chemical Society Reviews, vol. 44, no. 7, pp. 1820-1835, 2015.

[8] P. Li, T. Li, Y. Zeng et al., "Biosynthesis of xanthan gum by Xanthomonas campestris LRELP-1 using kitchen waste as the sole substrate," Carbohydrate Polymers, vol. 151, pp. 684-691, 2016.

[9] V. Pegos, R. Canevarolo, A. Sampaio, A. Balan, and A. Zeri, "Xanthan Gum Removal for 1H-NMR Analysis of the Intracellular Metabolome of the Bacteria Xanthomonas axonopodis pv. citri 306," Metabolites, vol. 4, no. 2, pp. 218-231, 2014.

[10] S. A. Rather, F. A. Masoodi, R. Akhter et al., "Application of guar-xanthan gum mixture as a partial fat replacer in meat emulsions," Journal of Food Science and Technology, vol. 53, no. 6, pp. 2876-2886, 2016.

[11] C. V. Nikiforidis and V. Kiosseoglou, "Physicochemical stability of maize germ oil body emulsions as influenced by oil body surface-xanthan gum interactions," Journal of Agricultural and Food Chemistry, vol. 58, no. 1, pp. 527-532, 2010.

[12] X. Chen, M. Wang, F. Yang, W. Tang, and X. Li, "Isolation and characterization of xanthan-degrading Enterobacter sp. nov. LB37 for reducing the viscosity of xanthan in petroleum industry," World Journal of Microbiology and Biotechnology, vol. 30, no. 5, pp. 1549-1557, 2014.

[13] J. F. Llamas-Moreno, L. M. Baiza-Durán, L. R. SaucedoRodríguez, and J. Félix Alaníz-De la O, "Efficacy and safety of chondroitin sulfate/xanthan gum versus polyethylene gly$\mathrm{col} /$ propylene glycol/hydroxypropyl guar in patients with dry eye," Clinical Ophthalmology, vol. 7, pp. 995-999, 2013.

[14] N. Vilardell, L. Rofes, V. Arreola, R. Speyer, and P. Clavé, "A Comparative Study Between Modified Starch and Xanthan Gum Thickeners in Post-Stroke Oropharyngeal Dysphagia," Dysphagia, vol. 31, no. 2, pp. 169-179, 2016.

[15] G. A. Sotiriou, A. Meyer, J. T. N. Knijnenburg, S. Panke, and S. E. Pratsinis, "Quantifying the origin of released $\mathrm{Ag}^{+}$ions from nanosilver," Langmuir, vol. 28, no. 45, pp. 15929-15936, 2012.

[16] T. M. Tolaymat, A. M. El Badawy, A. Genaidy, K. G. Scheckel, T. P. Luxton, and M. Suidan, "An evidence-based environmental perspective of manufactured silver nanoparticle in syntheses and applications: a systematic review and critical appraisal of peer-reviewed scientific papers," Science of the Total Environment, vol. 408, no. 5, pp. 999-1006, 2010.

[17] S. Chernousova and M. Epple, "Silver as antibacterial agent: ion, nanoparticle, and metal," Angewandte Chemie International Edition, vol. 52, no. 6, pp. 1636-1653, 2013.

[18] S. Iravani, "Synthesis of silver nanoparticles: chemical, physical and biological methods," Research in Pharmaceutical Sciences, vol. 9, no. 6, pp. 385-406, 2014.

[19] A. Taglietti, Y. A. Diaz Fernandez, E. Amato et al., "Antibacterial activity of glutathione-coated silver nanoparticles against gram positive and gram negative bacteria," Langmuir, vol. 28, no. 21, pp. 8140-8148, 2012.

[20] P. Pallavicini, C. R. Arciola, F. Bertoglio et al., "Silver nanoparticles synthesized and coated with pectin: An ideal compromise for anti-bacterial and anti-biofilm action combined with wound-healing properties," Journal of Colloid and Interface Science, vol. 498, pp. 271-281, 2017.

[21] S. Zhang, Y. Guo, Y. Dong et al., "A Novel Nanosilver/Nanosilica Hydrogel for Bone Regeneration in Infected Bone Defects," ACS Applied Materials and Interfaces, vol. 8, no. 21, pp. 13242-13250, 2016.

[22] Y. He, Z. Du, S. Ma et al., "Biosynthesis, Antibacterial Activity and Anticancer Effects Against Prostate Cancer (PC-3) Cells of Silver Nanoparticles Using Dimocarpus Longan Lour. Peel Extract," Nanoscale Research Letters, vol. 11, no. 1, article 300, 2016.

[23] S. J. Jang, I. J. Yang, C. O. Tettey, K. M. Kim, and H. M. Shin, "In-vitro anticancer activity of green synthesized silver nanoparticles on MCF-7 human breast cancer cells," Materials Science and Engineering C, vol. 68, pp. 430-435, 2016.

[24] N. Reddy and Y. Yang, "Citric acid cross-linking of starch films," Food Chemistry, vol. 118, no. 3, pp. 702-711, 2010.

[25] N. E. El-Naggar and N. A. M. Abdelwahed, "Application of statistical experimental design for optimization of silver nanoparticles biosynthesis by a nanofactory Streptomyces viridochromogenes," Journal of Microbiology, vol. 52, no. 1, pp. 5363, 2014.

[26] P. Lodeiro, E. P. Achterberg, J. Pampín, A. Affatati, and M. S. El-Shahawi, "Silver nanoparticles coated with natural polysaccharides as models to study AgNP aggregation kinetics using UV-Visible spectrophotometry upon discharge in complex environments," Science of the Total Environment, vol. 539, pp. 7-16, 2016.

[27] W. Pu, Y. Su, J. Li et al., "High incidence of oxacillin-susceptible mecA-positive Staphylococcus aureus (OS-MRSA) associated with bovine mastitis in China," PLoS ONE, vol. 9, no. 2, Article ID e88134, 2014.

[28] G. A. Sotiriou and S. E. Pratsinis, "Antibacterial activity of nanosilver ions and particles," Environmental Science and Technology, vol. 44, no. 14, pp. 5649-5654, 2010.

[29] K. I. Batarseh, "Anomaly and correlation of killing in the therapeutic properties of siliver (I) chelation with glutamic and tartaric acids," Journal of Antimicrobial Chemotherapy, vol. 54, no. 2, pp. 546-548, 2004.

[30] M. J. Piao, K. A. Kang, I. K. Lee et al., "Silver nanoparticles induce oxidative cell damage in human liver cells through inhibition of reduced glutathione and induction of mitochondriainvolved apoptosis," Toxicology Letters, vol. 201, no. 1, pp. 92100, 2011.

[31] N. R. Chowdhury, M. MacGregor-Ramiasa, P. Zilm, P. Majewski, and K. Vasilev, “'Chocolate' silver nanoparticles: Synthesis, antibacterial activity and cytotoxicity," Journal of Colloid and Interface Science, vol. 482, pp. 151-158, 2016.

[32] D. Paredes, C. Ortiz, and R. Torres, "Synthesis, characterization, and evaluation of antibacterial effect of Ag nanoparticles against escherichia coli O157:H7 and methicillin-resistant staphylococcus aureus (MRSA)," International Journal of Nanomedicine, vol. 9, no. 1, pp. 1717-1729, 2014.

[33] C. M. Murphy and F. J. O'Brien, "Understanding the effect of mean pore size on cell activity in collagen-glycosaminoglycan scaffolds," Cell Adhesion and Migration, vol. 4, no. 3, pp. 377381, 2010.

[34] N. Huebsch, E. Lippens, K. Lee et al., "Matrix elasticity of voidforming hydrogels controls transplanted-stem-cell-mediated bone formation," Nature Materials, vol. 14, no. 12, pp. 1269-1277, 2015. 
[35] H. M. Lappin-Scott and C. Bass, "Biofilm formation: attachment, growth, and detachment of microbes from surfaces," American Journal of Infection Control, vol. 29, no. 4, pp. 250-251, 2001.

[36] C. R. Arciola, D. Campoccia, P. Speziale, L. Montanaro, and J. W. Costerton, "Biofilm formation in Staphylococcus implant infections. A review of molecular mechanisms and implications for biofilm-resistant materials," Biomaterials, vol. 33, no. 26, pp. 5967-5982, 2012.

[37] Y. Deng, J. Ren, G. Chen et al., "Evaluation of polypropylene mesh coated with biological hydrogels for temporary closure of open abdomen," Journal of Biomaterials Applications, vol. 31, no. 2, pp. 302-314, 2016. 

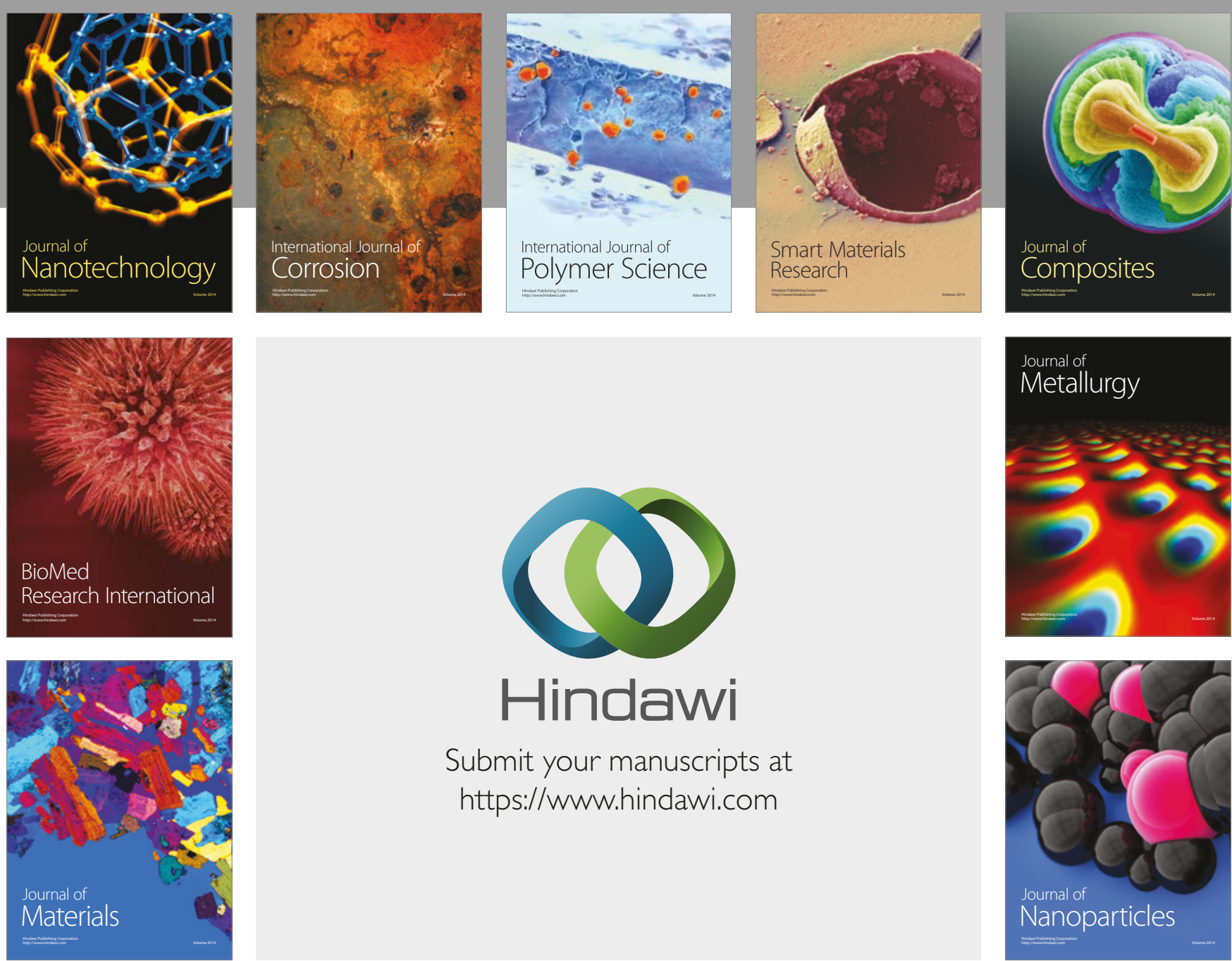

\section{Hindawi}

Submit your manuscripts at

https://www.hindawi.com
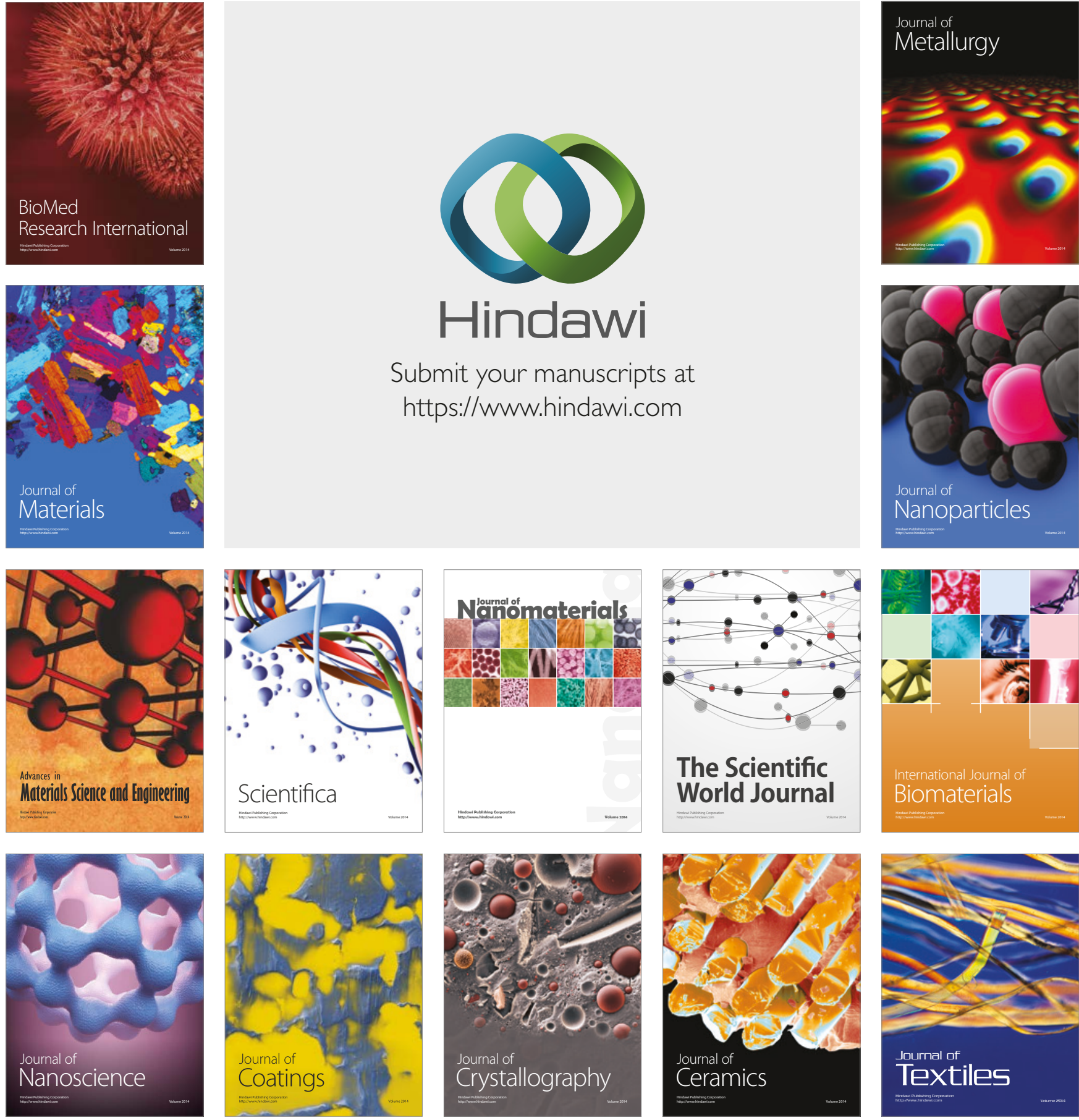

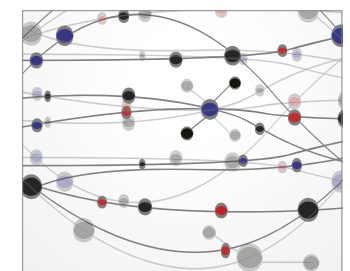

The Scientific World Journal
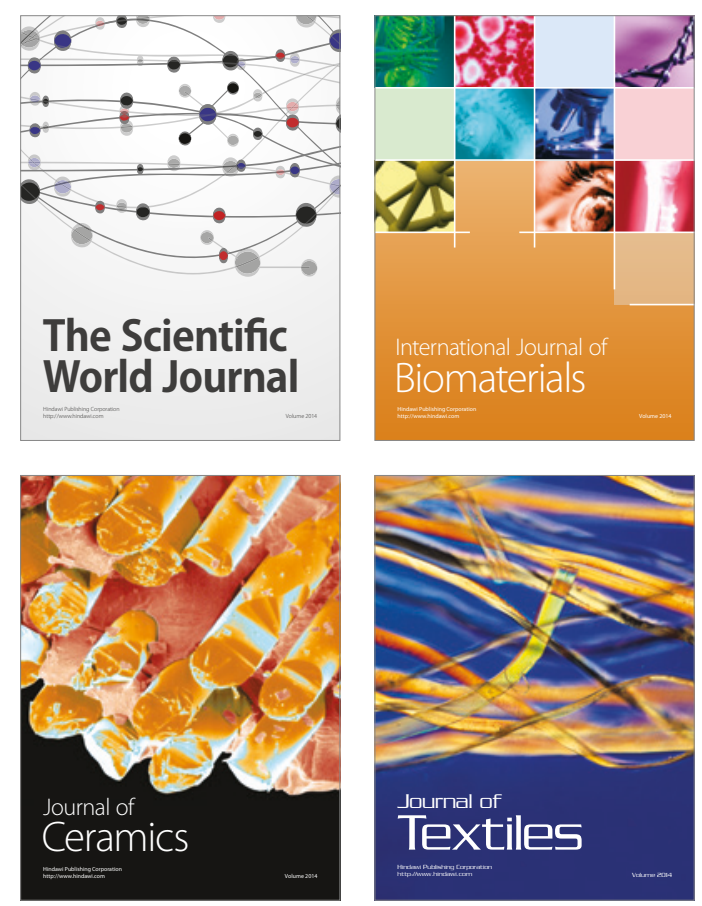